\title{
Ribonucleotide reductase subunit M1 is a possible chemoresistance marker to gemcitabine in biliary tract carcinoma
}

\author{
KAZUMA OHTAKA, NAOHIKO KOHYA, KEN SATO, YOSHIHIKO KITAJIMA, \\ TAKAO IDE, MAYUMI MITSUNO and KOHJI MIYAZAKI
}

Department of Surgery, Saga University Faculty of Medicine, Saga, Japan

Received February 22, 2008; Accepted April 29, 2008

DOI: $10.3892 /$ or_00000004

\begin{abstract}
Biliary tract carcinoma is a relatively rare tumor with a poor prognosis. Surgical resection is the only potential cure. However, the efficacy of chemotherapeutic agents is disappointing in advanced or recurrent cases. Gemcitabine (GEM) appears to be one of the most promising chemotherapeutic agents in biliary tract carcinoma, and ribonucleotide reductase subunit M1 (RRM1) plays an important role in GEM resistance. The aim of this study was to evaluate the correlation between the expression of RRM1 and the response to GEM in biliary tract carcinoma in vitro and in vivo. The drug sensitivity to GEM was assessed by MTT assay. The expression of RRM1 was evaluated by quantitative RT-PCR, a Western blot analysis and immunohistochemistry. RNAi assay was used to investigate the down-regulation of the expression of RRM1. After the RRM1-specific RNAi transfection, a cell growth assay was performed to evaluate the drug sensitivity to GEM, and flow cytometry and TUNEL assay were performed to evaluate apoptosis. The results showed that in 6 biliary tract carcinoma cell lines, a tendency for a positive correlation between the expression of RRM1 and $\mathrm{IC}_{50}$ for GEM exists $(\mathrm{R}=0.620, \mathrm{p}=0.19)$. The transfection of the RRM1-specific RNAi suppressed the expression level of RRM1 in a dose-dependent manner. After the transfection of RRM1-specific RNAi into G-415, the drug sensitivity to GEM markedly increased $(\mathrm{p}<0.001)$, and apoptosis was highly induced according to flow cytometry and the TUNEL assay. In an analysis of cancer tissue specimens obtained from the 12 patients treated with GEM for biliary tract cancer, RRM1 immunostaining was strongly positive in all of the PD cases (3/3), while weakly positive in all of the PR cases except for one (4/5). In conclusion, RRM1
\end{abstract}

Correspondence to: Dr Kohji Miyazaki, Department of Surgery, Saga University Faculty of Medicine, 5-1-1 Nabeshima, Saga 849-8501, Japan

E-mail: miyazak2@cc.saga-u.ac.jp

Key words: ribonucleotide reductase subunit M1, gemcitabine, biliary tract carcinoma, drug sensitivity may be one of the key marker molecules for GEM chemotherapy that overcomes advanced and recurrent biliary tract carcinoma.

\section{Introduction}

Biliary tract carcinoma, which consists of gallbladder carcinoma and cholangiocarcinoma, is a relatively rare tumor (1-3). The mortality rate of this cancer has been increasing worldwide over the past few decades, and the prognosis is poor $(1,2)$. The mortality rates are highest among Native American Indian women from the Southwestern United States, and among Chilean and Japanese women (4). The 5-year survival rate is $<25 \%$ for intra-, and extrahepatic bile duct carcinoma and $61 \%$ for gallbladder carcinoma, even after a radical resection of the tumor $(1,2,5)$. Surgical resection remains the mainstay of treatment and offers the only potential cure.

The role of chemotherapy remains controversial in biliary tract carcinoma. The efficacy of chemotherapy for advanced disease is relatively limited, with a response rate of $<10 \%$ for single-agent fluorouracil, and $<17 \%$ for a fluorouracil-based combination (6-10). Among the chemotherapeutic agents, gemcitabine (GEM) appears to be one of the most promising. Reports have described the efficacy of the single-agent GEM, with a response rate of $\sim 30 \%$ and a median survival time of $\sim 15$ months. Phase II investigations into a GEM-based combination have increased $(11,12)$. Chemotherapy resistance is mainly determined by tumor cell resistance to chemotherapeutic agents, which is either acquired or naturally present (13).

Various investigations have been conducted in order to detect the molecular markers of GEM that are involved in cell cycle regulation, proliferation or apoptosis, such as p53, Bcl-xl, c-Src, BNIP3 and focal adhesion kinase (14-18). Several studies have suggested that the deoxycytidine kinase (dCK), cytidine deaminase (CDA), rebonucleotide reductase subunit M1 (RRM1), rebonucleotide reductase subunit M2 (RRM2) and p53R2 play an important role in the GEM resistance of various cancers as metabolic enzymes of the drug (19-25).

RRM1 is a member of human ribonucleotide reductase (RR), consisting of RRM1, RRM2 and p53R2 (26,27). RR acts as the rate-limiting enzyme in de novo DNA synthesis and converts ribonucleotides to deoxyribonucleotide. This 
conversion is necessary for DNA polymerization and repair $(28,29)$. GEM is a deoxycytidine analog, which is initially phosphorylated by the deoxycytidine kinase to gemcitabine monophosphate. Subsequent phosphorylation steps yield gemcitabine diphosphate and triphosphate. Gemcitabine diphosphate inhibits RR, decreasing the cellular pool of deoxycitidine triphosphate that competes with gemcitabine triphosphate for incorporation into DNA. The incorporation of gemcitabine triphosphate into DNA inhibits replication, with the subsequent induction of apoptosis $(30,31)$. Although the RR enzymatic activity is modulated by the levels of RRM2 and p53R2, RRM1 is believed to be a key molecule for GEM resistance in vitro and in vivo (21-23,32,33).

GEM has been formally approved for the treatment of biliary tract carcinoma as a chemotherapeutic agent by medical insurance in Japan. The efficacy of GEM is expected in advanced or recurrent biliary tract carcinoma.

However, to our knowledge no reports exist which describe the correlation between the expression of RRM1 and the chemosensitivity to GEM in biliary tract carcinoma. Thus, we analyzed the correlation between the expression of RRM1 and the chemosensitivity to GEM in biliary tract carcinoma in vitro and investigated the effect on the sensitivity to GEM by RRM1 deficiency using RNAi.

Furthermore, we investigated the expression of RRM1 using 47 surgically resected biliary tract carcinoma specimens by immunohistochemistry to determine the percentage of possible candidates, and examined for any correlations between the expression of RRM1 and the response to GEM in 12 patients who had been treated with GEM.

\section{Materials and methods}

Cell lines and culture. Four gallbladder carcinoma cell lines (GB-d1, GBK-1, KMG-C and G-415) and two biliary tract carcinoma cell lines (HBDC and TFK-1) were used. GB-d1 was provided by Dr T. Date (Department of Surgery I, Kyushu University, Fukuoka, Japan). GBK-1 was provided by Dr H. Egami (Department of Gastroenterological Surgery, Kumamoto University, Kumamoto, Japan). KMG-C was provided by Dr H. Yano (Department of Pathology, Kurume University, Kurume, Japan). G-415 was provided by Dr S. Koyama (Department of Internal Medicine, Tsukuba University, Tsukuba, Japan). HBDC was established in our laboratory (W.J.) (34). TFK-1 was from the Cell Resource Center for Biomedical Research, Tohoku University (Miyagi, Japan). The cell lines were cultured in RPMI-1640 (Nissui Pharmaceutical, Tokyo, Japan) supplemented with $10 \%$ inactivated FBS (Biofluids, MD, USA) and $100 \mu \mathrm{g} / \mathrm{ml}$ kanamycin. The cell lines were incubated at $37^{\circ} \mathrm{C}$ in a humidified atmosphere of $5 \% \mathrm{CO}_{2}$ in air.

Drugs. GEM was provided by Eli Lilly and Co. (Indianapolis, IN, USA). It was dissolved in $\mathrm{dH}_{2} \mathrm{O}$ and diluted in RPMI-1640 medium to the required concentrations immediately before use.

MTT assay. The drug sensitivity of GEM was analyzed by MTT assay using a CellTiter 96 non-radioactive cell proliferation assay kit (Promega, Madison, WI, USA). In brief, $1 \times 10^{4}$ cells of each cell line/well were seeded in triplicate onto 96-well plates. After $24 \mathrm{~h}$, the cells were exposed for $72 \mathrm{~h}$ to GEM at a concentration ranging from $100 \mathrm{nM}$ to $10 \mathrm{mM}$. The proliferation curves were then constructed by calculating the mean value of the optical density measurements at $590 \mathrm{~nm}$ using a 96-well plate reader (Immuno-mini NJ2300, Nalge Nunc International K.K., Tokyo, Japan).

Quantitative RT-PCR. Total RNA was extracted from each of the cell lines using Isogen (Nippongene, Toyama, Japan). For each cell line, $1 \mu \mathrm{g}$ of extracted total RNA was subjected to reverse transcription reaction, using an RNA LA PCR ${ }^{\mathrm{TM}}$ kit (AMV) version.1.1 (Takara Biomedicals, Tokyo, Japan). The cDNA was used as a template. The RRM1 mRNA level was quantified by a LightCycler (Roche Diagnostics, Mannheim, Germany), using a LightCycler ${ }^{\circledR}$ FastStart DNA Master SYBR-Green I (Roche Diagnostics). The details of the primers of RRM1 used in this study were: RRM1 forward, 5'-GGA GGA ATT GGT GTT GCT GT-3' and RRM1 reverse, 5'-GCT GCT CTT CCT TTC CTG TG-3'. The PCR conditions were: initial denaturation at $95^{\circ} \mathrm{C}$ for $3 \mathrm{~min}$, followed by 50 cycles of $15 \mathrm{sec}$ denaturation at $95^{\circ} \mathrm{C}, 5 \mathrm{sec}$ annealing at $60^{\circ} \mathrm{C}$ and $10 \mathrm{sec}$ extension at $72^{\circ} \mathrm{C}$. The expression level of RRM1 mRNA was adjusted by the level of $\beta$-actin mRNA, and expressed as a ratio to $\beta$-actin mRNA. This analysis was performed in triplicate and the mean values were calculated.

Transient transfection of RRM1-specific RNAi and protein extraction. The RRM1-specific and control RNAi were designed by iGene Therapeutics, Inc. (Tsukuba, Japan). The RRM1-specific RNAi was: sense 5'-UGC ACA GAA AUA GUG GAG UAC ACC AAG-3' and anti-sense 5'-UAA CGU GUC UUU AUC ACC UCA UGU GGU-3'. RNAi was dissolved in TE buffer to make a $100 \mu \mathrm{M}$ stock. G-415 cells $\left(1.5 \times 10^{4}\right)$ were seeded onto 6-well plates. After $24 \mathrm{~h}$, the growth medium was removed, the cells were washed three times with phosphate buffered saline (PBS), and $800 \mu 1$ medium without serum were added to each well containing cells. Transfection was performed using Oligofectamine ${ }^{\mathrm{TM}}$ reagent (Invitrogen, Carlsbad, CA, USA) following the Invitrogen protocol. After $48 \mathrm{~h}$ of transfection, the cultured cells were harvested using $0.05 \%$ trypsin, washed twice in PBS, homogenized in a lysis buffer composed of $300 \mathrm{mM}$ $\mathrm{NaCl}, 50 \mathrm{mM}$ Tris-Hcl (pH 7.6), 0.5\% Triton X-100, and a protease inhibitor cocktail mix (Roche Diagnostics), and put on ice for $1 \mathrm{~h}$. After centrifugation at 15,000 rpm for $20 \mathrm{~min}$, the supernatants were collected and the protein concentration was equalized in each sample.

Western blot analysis. The samples were dissolved in NuPage $^{\text {TM }}$ LDS sample buffer (Invitrogen, CA, USA) and $1 \mathrm{M}$ DTT, and heated for $5 \mathrm{~min}$ at $95^{\circ} \mathrm{C}$. The samples were subjected to NuPage 4-12\% Bis-Tris Gel (Invitrogen) and electrophoretically transferred onto a Hybond ${ }^{\mathrm{TM}}$ nitrocellulose enhanced chemiluminescence membrane (Amersham Pharmacia Biotech, Buckinghamshire, UK). After blocking with $5 \%$ non-fat milk for $1 \mathrm{~h}$, the membrane was incubated with primary antibodies overnight at $4^{\circ} \mathrm{C}$. The primary antibodies used in the Western blot analysis were anti-RRM1 (1:500, Chemicon, CA, USA), and anti- $\beta$-actin ( $1: 1000$ Sigma, MO, USA). After incubation with the corresponding 
secondary antibodies, the signals were finally developed using an enhanced chemiluminescence detection kit (Amersham Pharmacia Biotech).

Cell growth assay. G-415 cells $\left(1 \times 10^{4}\right)$ were seeded onto 6-well plates in triplicate. After $24 \mathrm{~h}$, the RRM1-specific and control RNAi were transfected following the described methods and the transfected cells were then cultured for $48 \mathrm{~h}$ in triplicate. The cells were then treated with $100 \mathrm{nM}$ GEM for $48 \mathrm{~h}$. Finally, the cells were harvested using Accutase (Innovative Cell Technologies Inc., San Diego, CA, USA) and the mean number for each group was calculated using the Burker-Turk hemocytometer.

Flow cytometry. The cell preparation was the same as described for the cell growth assay. The cells were harvested using Accumax (Innovative Cell Technologies Inc.) and washed twice with PBS. The cells were fixed in $70 \%$ ethanol at $4^{\circ} \mathrm{C}$, and stored at $-20^{\circ} \mathrm{C}$ until analysis. The ethanol was removed, and the cells were stained with a propidium iodide solution $(50 \mu \mathrm{g} / \mathrm{ml}$ in PBS with $100 \mathrm{U} / \mathrm{ml}$ RNase) for $30 \mathrm{~min}$ at room temperature in the dark. The samples were analyzed using the FACSCalibur and CellQuest software program version 2.0 (Becton-Dickinson, Franklin Lakes, NJ, USA).

TUNEL assay. The number of $4 \times 10^{3} \mathrm{G}-415$ parent cells for the RRM1-specific or control RNAi transfection was seeded onto the Lab-Tek Chamber Slide system (Nalge Nunc International K.K.). After 24 h, the RRM1-specific and control RNAi were transfected following the described methods and the transfected cells were cultured for $24 \mathrm{~h}$. Then, the cells were treated with $100 \mathrm{nM}$ GEM for $24 \mathrm{~h}$. Finally, we tried to detect the apoptotic cells using the TACS ${ }^{\text {TM }}$ TdT kit (R\&D Systems, Minneapolis, MN, USA) following the R\&D Systems protocol. The presence of apoptotic change was defined as brown (dark) cells in each case (four visual fields $\mathrm{x} 300$ fields). The data are shown as the mean \pm SD.

Clinical samples. Resected specimens were obtained from 47 patients with biliary tract carcinoma who underwent a surgical resection at our institution from January 2000 to December 2006. All of the patients gave their informed consent to fully participate in this study. The samples were embedded in paraffin after surgical resection. Twenty-four cases were gallbladder carcinoma and 23 cases were bile duct carcinoma. Twelve patients had a measurable metastatic or recurrent lesion and were treated with GEM. The response to GEM was defined as: complete response (CR), partial response (PR), stable disease (SD) and progressive disease (PD). This classification was based on the New guidelines to evaluate the response to treatment in solid tumors (RECIST guidelines) (35).

Immunohistochemistry. Immunohistochemical staining was performed according to the procedures of a previous report (36). For antigen retrieval, the specimens were treated with microwave boiling. Thereafter, the slides were incubated in $3 \%$ hydrogen peroxide for $10 \mathrm{~min}$ to block the intrinsic peroxidase activity. The primary antibodies were placed onto the slides which were incubated at room temperature for $2 \mathrm{~h}$.
A

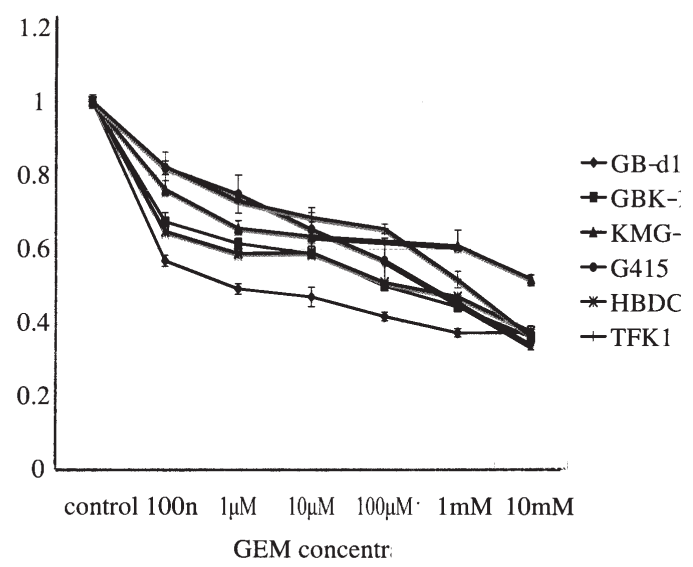

B

$\mathrm{IC}_{50}$ for $\mathrm{GEM}$

\begin{tabular}{|c|c|}
\hline Cell lines & $\mathrm{IC}_{50}(\mathrm{uM})$ \\
\hline GB-d 1 & 0.98 \\
\hline GBK-1 & 99.17 \\
\hline KMG-C & 11972.38 \\
\hline G-415 & 3151.17 \\
\hline HBDC & 305.27 \\
\hline TFK-1 & 2003.24 \\
\hline
\end{tabular}

Figure 1. (A) Drug sensitivity to GEM in 6 biliary tract carcinoma cell lines. The cells were treated with various concentrations of GEM for $72 \mathrm{~h}$. The proliferation curves were estimated by MTT assay and constructed by calculating the mean value of the optical density measurements at $590 \mathrm{~nm}$ using a 96-well plate reader. (B) The $\mathrm{IC}_{50}$ for GEM in 6 biliary tract carcinoma cell lines estimated by MTT assay.

The primary antibody used in immunohistochemistry was the anti-RRM1 antibody (1:50 dilution, Chemicon). After washing, the slides were incubated in a secondary antibody for $30 \mathrm{~min}$ at room temperature. Then the slides were washed and incubated in a chromogen solution from a liquid DAB (3,3-diaminobenzidine) substrate kit (Nichirei Co., Tokyo, Japan). Finally, the slides were counterstained with hematoxylin.

The stained sections were evaluated in a blind manner without clinical information by two investigators, including one pathologist. The plasma and stroma cells showed a weakly positive RRM1 staining for cytoplasm, which was considered as an internal control. The cells that showed stronger staining or the same staining compared to the plasma and stroma cells for cytoplasm were regarded as strongly positive, and the cells that showed weaker staining than the plasma and stroma cells for cytoplasm were regarded as weakly positive.

Statistical analysis. The relationships among $\mathrm{IC}_{50}$ for $\mathrm{GEM}$ and the expression of RRM1 in the 6 biliary tract carcinoma cell lines were assessed statistically by Pearson's correlation. The GEM sensitivity and apoptotic index after RNAi transfection in the G-415 cell line was assessed statistically by the Bonferroni/Dunn test. The statistical analyses were performed using the Statview version 5.0 software program (SAS Institute Inc. Cary, NC, USA). 
A
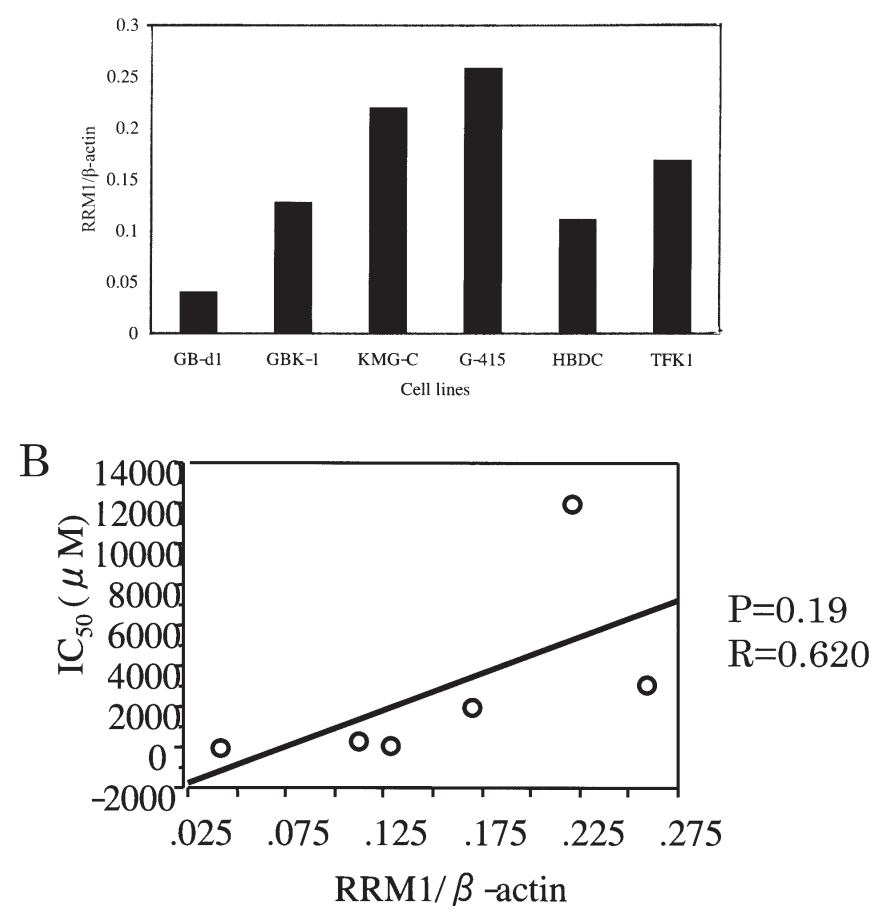

Figure 2. (A) The expression of RRM1 mRNA in 6 biliary tract carcinoma cell lines was calculated by quantitative RT-PCR. The expression level of RRM1 mRNA was adjusted by the level of $\beta$-actin mRNA, and expressed as a ratio to $\beta$-actin mRNA. This analysis was performed in triplicate and the mean values were calculated. (B) The relationship between the expression level of RRM1 and $\mathrm{IC}_{50}$ for GEM in 6 biliary tract carcinoma cell lines. A positive correlation between the expression of RRM1 mRNA and $\mathrm{IC}_{50}$ for GEM in the six cell lines was observed $(\mathrm{R}=0.620)$, although this correlation was not statistically significant $(\mathrm{p}=0.19)$.

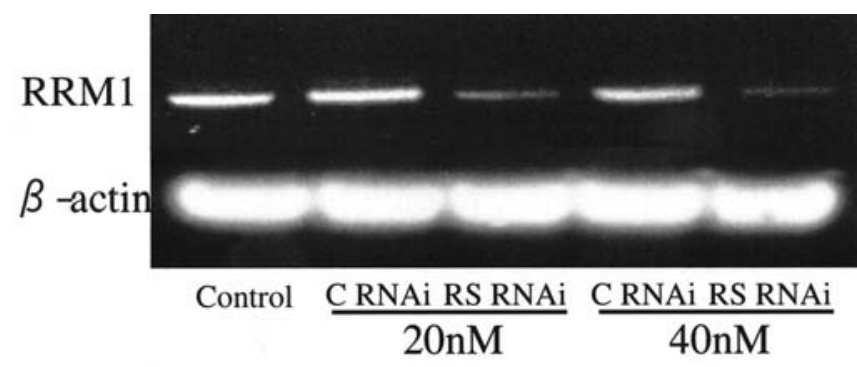

Figure 3. (A) Western blot analysis of the RRM1 expression after 20 or $40 \mathrm{nM}$ RRM1-specific RNAi transfection (C RNAi, control RNAi and RS RNAi, RRM1-specific RNAi).

\section{Results}

Drug sensitivity to GEM in the 6 biliary tract carcinoma cell lines. The drug sensitivity to GEM was assessed by MTT assay. The proliferation curves of the six cell lines are shown in Fig. 1A. GB-d1 was the most sensitive to GEM. In contrast, KMG-C was the most resistant. Each $\mathrm{IC}_{50}$ for $\mathrm{GEM}$ in the six cell lines is shown in Fig. 1B. The $\mathrm{IC}_{50}$ for GEM in the KMG-C cell line was $\sim 12,000$-fold higher than in the GB-d1 cell line.

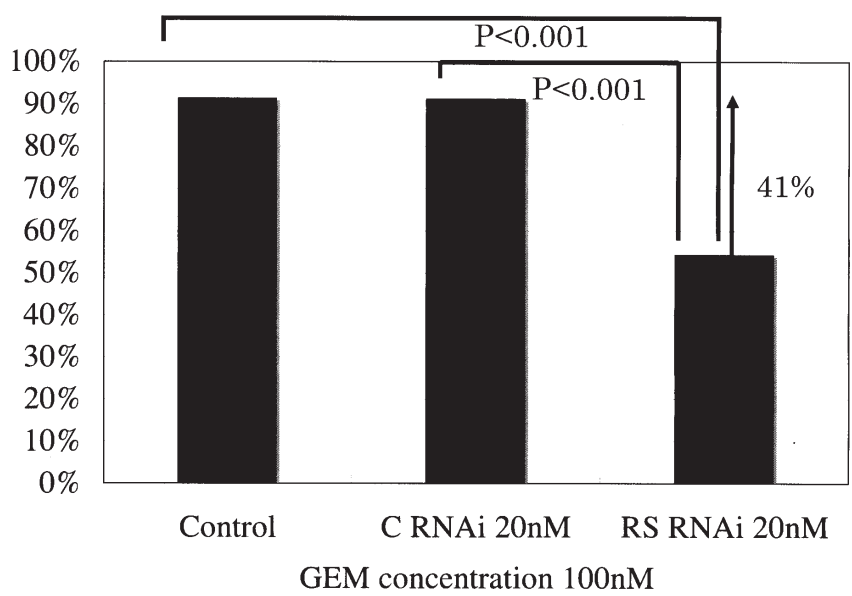

Figure 4. Drug sensitivity to GEM after RRM1-specific RNAi transfection in the G-415 cell line. The rate of cell growth was estimated as the number of cells with treatment divided by the number of cells without treatment in each group: (A) control, (B) control RNAi transfection and (C) RRM1-specific RNAi transfection.

Relationship between the expression of RRM1 mRNA and the $I C_{50}$ for GEM in the 6 biliary tract carcinoma cell lines. The expression of RRM1 mRNA in the 6 biliary tract cell lines was assessed by quantitative RT-PCR, and the result is shown in Fig. 2A. The expression of RRM1 mRNA in G-415 was the highest among the six cell lines. In contrast, that of GB-d1 was the lowest and most sensitive to GEM. The relationship between the expression of RRM1 mRNA and $\mathrm{IC}_{50}$ for GEM in the six cell lines is shown in Fig. 2B. The result shows a tendency for a positive correlation between the expression of RRM1 mRNA and $\mathrm{IC}_{50}$ for GEM in the six cell lines $(\mathrm{R}=0.620)$, although this correlation was not statistically significant $(\mathrm{p}=0.19)$.

Down-regulation of RRMI expression by RNAi. To downregulate the expression of RRM1, the RRM1-specific RNAi was transfected into the G-415 cell line. The ability of RNAi to down-regulate the expression of RRM1 was analyzed by Western blot analysis (Fig. 3). The expression of RRM1 was significantly down-regulated by the RRM1-specific RNAi transfection at $48 \mathrm{~h}$, compared to the G-415 parent cell line and the control RNAi. The RRM1-specific RNAi repressed RRM1 expression in G-415 in a dose-dependent manner.

Drug sensitivity to GEM after RRMI-specific RNAi transfection. To analyze whether or not the drug sensitivity to GEM increases after the down-regulation of RRM1, we performed a cell growth assay. The RRM1-specific RNAi (20 $\mathrm{nM}$ ) or control RNAi $(20 \mathrm{nM})$ were transiently transfected into G-415. After $48 \mathrm{~h}$ of transfection, the cells with and without transfection were treated with $100 \mathrm{nM}$ GEM for $48 \mathrm{~h}$. The cells were harvested and counted, and compared with the cells without GEM treatment. Fig. 4 shows the rate of cell growth after $100 \mathrm{nM}$ of GEM treatment. In the RRM1-specific RNAi transfection group, the rate of cell growth decreased to 59\%, compared to the control RNAi transfection and the group without transfection. This result 

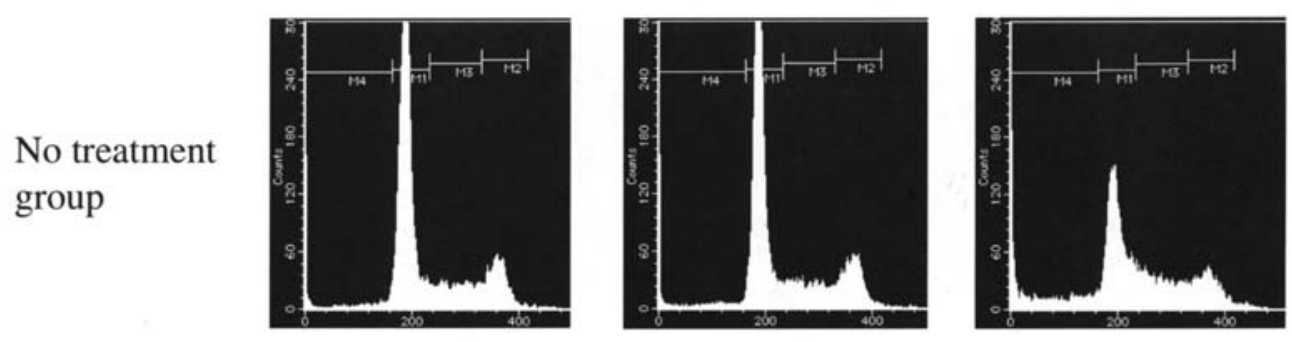

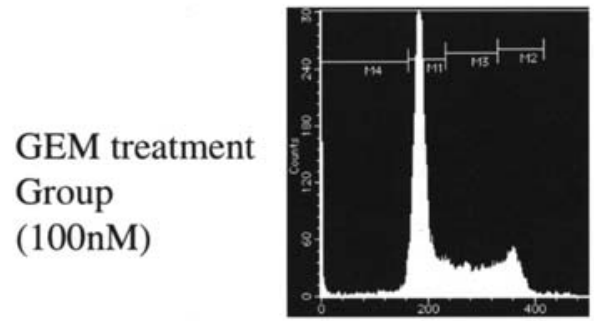

Control

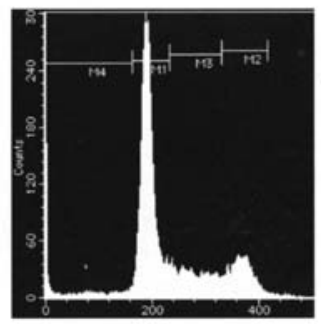

C RNAi

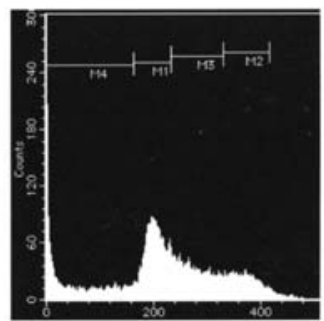

RS RNAi

Figure 5. A cell cycle analysis of GEM after RNAi transfection in the G-415 cell line. The concentration of GEM was $100 \mathrm{nM}$. The samples were analyzed using FACSCalibur (counting 15,000 cells per sample) and the CellQuest software program version 2.0.
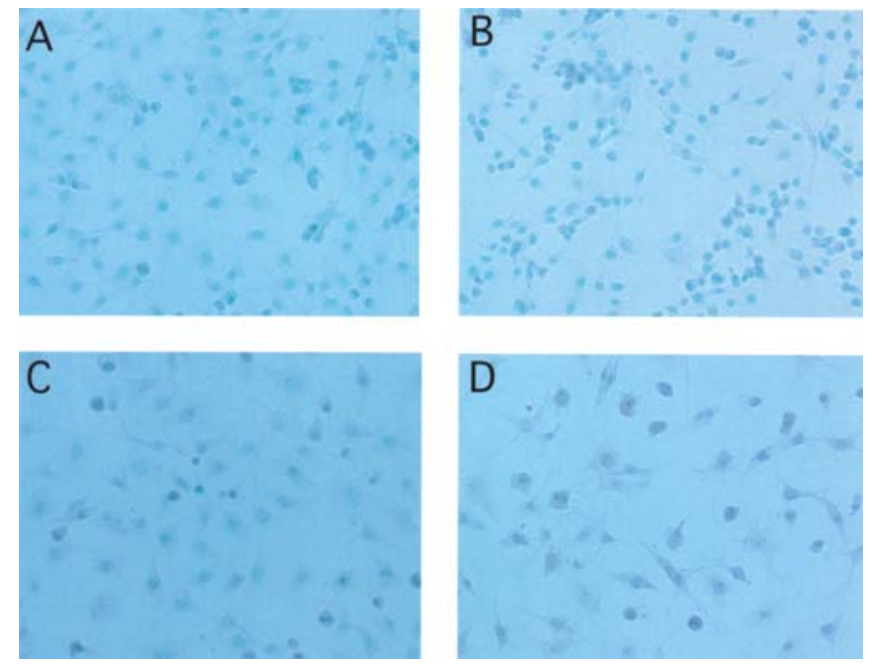

Figure 6. TUNEL assay for G-415 cells. The specimens were displayed at high power field (x400). The apoptotic cells were darkly stained by DAB. (A) control, (B) RRM1-specific RNAi-transfected cells, (C) control with GEM $100 \mathrm{nM}$ treatment and (D) RRM1-specific RNAi-transfected cells with GEM $100 \mathrm{nM}$ treatment.

indicates that the suppression of RRM1 expression increases drug sensitivity to GEM.

Flow cytometry after RNAi transfection with or without GEM treatment. Fig. 5 shows the cell fractions of the G-415 cells, which were either the transfected control or RRM1-specific RNAi with or without GEM treatment. Table I shows the detailed fraction of each group. In the groups without treatment, there was no significant difference in the G-415 cell fraction between the group without transfection and the control
RNAi transfection group. In contrast, there were increases in the sub G1 and S phase fractions in the RRM1-specific RNAi transfection group. In the groups with GEM treatment, there was no significant change in the G-415 cell fraction without transfection and with control RNAi transfection. However, there was a marked increase in the sub G1 phase fraction in the RRM1-specific RNAi transfection group. Furthermore, in the group with RRM1-specific RNAi transfection, an increase of $>10 \%$ was observed in the sub G1 phase fraction by the GEM treatment group compared to the non-treatment group.

Apoptotic change by GEM after RRMI-specific RNAi transfection. In the case without treatment, there were a small number of apoptotic cells, and no significant difference between the without transfection group and the RRM1specific RNAi transfection group. However, in the RRM1specific transfection group, a large number of apoptotic cells were observed with $100 \mathrm{nM}$ of GEM treatment. There was a significant difference in the apoptotic change between the group without transfection and with the RRM1-specific RNAi transfection group (Figs. 6 and 7).

Examination of the correlation between the expression of RRM1 and the response to GEM in clinical cases. The expression of RRM1 in clinical cases was examined by immunohistochemistry using surgically resected specimens. RRM1 staining was localized in the cytoplasm. In 47 clinical cases, 29 were strongly positive, while $18(38 \%)$ were weakly positive (Fig. 8). We analyzed the correlation between the expression of RRM1 and the response to GEM. Twelve patients had been treated with GEM and the response to GEM was: CR ( $n=0), \operatorname{PR}(n=5), \operatorname{SD}(n=4)$ and PD $(n=3)$, respectively. As a result, the RRM1 staining was strongly positive in the PD cases (3/3), while the RRM1 staining was 


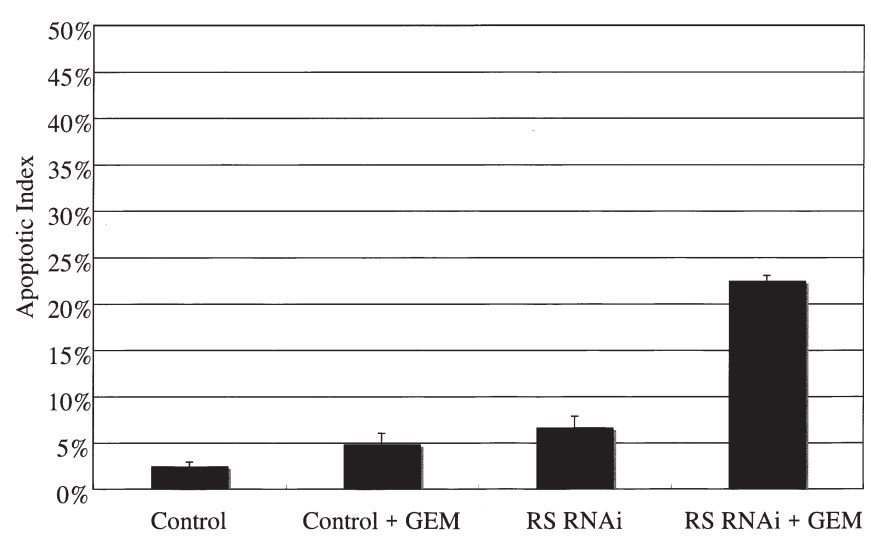

Figure 7. The apoptotic index after the TUNEL assay in the G-415 cell line. The apoptotic cells were calculated by scoring the dark staining cells in each of the specimens ( 4 visual fields, $x 400$ field). The data are shown as the mean \pm SD. Statistical significance was determined using the Bonferroni/ Dunn test. The apoptotic index was estimated as the ratio of apoptotic to total cell number.

weakly positive the PR cases except for one (4/5). The correlation between the expression of RRM1 and the response to GEM is summarized in Table II.

\section{Discussion}

Biliary tract carcinoma is a relatively rare tumor, in which surgery brings the only potential cure $(1,2,5)$. However, even resection has shown a dismal outcome in the advanced stage of carcinoma and the efficacy of the conventional chemotherapy has been disappointing. Among the recently developed chemotherapeutic agents, GEM appears to be one of the most promising. Several reports have described a marked improvement in the efficacy of GEM alone and GEM-based combination chemotherapy $(11,12)$. Various investigations have been conducted to detect the molecular marker of GEM, that involved RR, which plays an important role in the GEM
Table I. Alteration of cell cycle fractions by GEM treatment after RNAi transfection.

\begin{tabular}{lcccc}
\hline & $\mathrm{G}_{1}(\%)$ & $\mathrm{S}(\%)$ & $\mathrm{G}_{2}-\mathrm{M}(\%)$ & sub $_{1}(\%)$ \\
\hline Control & 61.48 & 15.05 & 14.64 & 8.94 \\
Control GEM & 55.79 & 18.56 & 13.12 & 12.75 \\
100 nM & & & & \\
CRNAi & 58.09 & 15.39 & 16.23 & 10.35 \\
C RNAi GEM & 55.19 & 16.57 & 14.91 & 13.18 \\
100 nM & & & & \\
RS RNAi & 34.93 & 19.22 & 13.58 & 31.84 \\
RS RNAi GEM & 25.92 & 19.27 & 11.35 & 42.86 \\
100 nM & & & & \\
\hline
\end{tabular}

Table II. Association between gemcitabine response and the expression of RRM1 in immunohistochemistry.

\begin{tabular}{lcc}
\hline Expression and response & $\begin{array}{c}\text { Strongly } \\
\text { positive }\end{array}$ & $\begin{array}{c}\text { Weakly } \\
\text { positive }\end{array}$ \\
\hline PR & 1 & 4 \\
SD & 2 & 2 \\
PD & 3 & 0 \\
\hline
\end{tabular}

resistance of various cancers as metabolic enzymes of the drug (14-25). RRM1 is a member of human RR, and RRM1 may be a key molecule to GEM according to previous reports (21-23).

However, there have been no reports examining RRM1 and the sensitivity to GEM in vitro and in vivo thus far. In the present study, we analyzed the correlation between the

Weak Positive
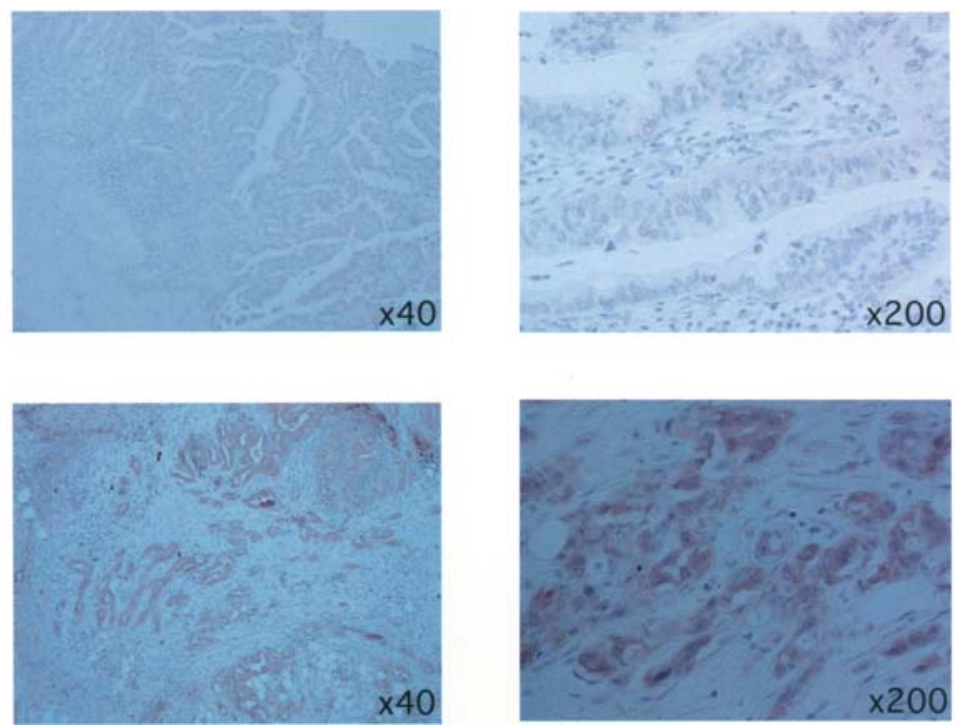

Figure 8. Immunohistochemical analysis of RRM1 using surgically resected specimens. RRM1 staining is seen in the cytoplasm. Definations: strongly positive, stronger staining than the plasma and stroma cells or the same staining as the plasma cells for cytoplasm; weakly positive, weaker staining than the plasma and stroma cells. 
expression of RRM1 and the chemosensitivity of GEM in biliary tract carcinoma.

We initially assessed the correlation between the expression of RRM1 and the chemosensitivity of GEM in 6 biliary tract carcinoma cell lines. The result showed a tendency for a positive correlation between the expression of RRM1 mRNA and $\mathrm{IC}_{50}$ for $\mathrm{GEM}$ in the six cell lines $(\mathrm{R}=0.620)$. These data were consistent with those of previous studies regarding the correlation between the expression of RRM1 and the chemosensitivity of GEM in non-small cell lung carcinoma, pancreas carcinoma and breast carcinoma cell lines, although this correlation was not statistically significant $(\mathrm{p}=0.19)$ $(21,23,36)$. These findings suggest that the RRM1 expression influences GEM sensitivity. However, a direct association of drug sensitivity to GEM with RRM1 expression still remains unclear.

We directly down-regulated the RRM1 expression using RRM1-specific RNAi demonstrating by Western blot analysis the expression of RRM1 to be significantly down-regulated.

We then analyzed the drug sensitivity of GEM in the G-415 cell line with or without RRM1 RNAi. The results showed that the drug sensitivity to GEM markedly increased with the down-regulation of RRM1 expression. This result suggested that RRM1 plays an important role in the drug sensitivity of GEM in the biliary tract carcinoma cell line. Notably, the cell proliferation in RRM1 down-regulation with only RRM1specific RNAi transfection without GEM treatment declined significantly (data not shown). We speculate that this repressed cell proliferation was due to the decreased DNA synthesis from the down-regulation of RRM1. However, Cao et al reported that the overexpression of RRM1 inhibited tumor growth in vitro and in vivo in colon cancer, and since RRM1 may be a putative tumor suppressor, RRM1 itself may be one of the main targets of gene therapy (37). The reason for this discrepancy is uncertain, but RRM1 showed no tumor suppressor activity in biliary tract carcinoma. Tissue specificity may exist in the RRM1 function.

We demonstrated how to influence the cell cycle by RRM1 down-regulation with GEM treatment in flow cytometry. There were increases in the sub G1 and S phase fractions in the RRM1-specific RNAi transfection. These results suggest that de novo DNA synthesis decreased due to the down-regulation of RRM1, which acts as a rate-limiting enzyme in de novo DNA synthesis. Therefore, cell cycle stops at the $\mathrm{S}$ phase and induces apoptosis. With GEM treatment, there were increases in the sub G1 phase fraction in the RRM1-specific RNAi transfection, and the increases were larger than in the RRM1-specific RNAi transfection without GEM treatment. We hypothesize that the cell cycle is stopped in the $\mathrm{S}$ phase after RRM1-specific RNAi transfection, and then GEM induces cells in the $S$ phase to apoptosis, because GEM has killing efficacy for cells in the $S$ phase (38).

To confirm this hypothesis, we analyzed the apoptotic index by TUNEL assay. The apoptotic index in the RRM1specific RNAi transfection with GEM treatment group was markedly higher than in the only RRM1-specific RNAitransfected group. This result partially supported the flow cytometry findings.
Finally, we analyzed the correlation between the expression of RRM1 and the drug sensitivity of GEM in clinical cases retrospectively. We demonstrated the expression of RRM1 by immunohistochemistry in surgically resected specimens. Of the cancers, $\sim 40 \%$ showed a repressed expression of RRM1, thereby suggesting that $40 \%$ of the patients are good candidates for GEM treatment. In 12 patients who had been treated with GEM, we showed that the biliary tract cancers with a strong expression of RRM1 were resistant to GEM, which supported the results obtained in the in vitro analysis. However, the number of subjects in this study was too small to conduct any comprehensive analysis. Further investigation and a large group of subjects are needed to prove this positive correlation.

In conclusion, we have demonstrated in this study that the expression of RRM1 should be a drug-sensitive marker of GEM for biliary tract carcinoma through in vitro study and clinical analysis. These results support the hypothesis that RRM1 may be one of the key molecules, which enable GEM chemotherapy to overcome advanced and recurrent biliary tract carcinoma.

\section{Acknowledgements}

We thank Dr K. Kai for his valuable advice on performing immunohistochemistry.

\section{References}

1. Henson DE, Albores-Saaverdra J and Corle D: Carcinoma of the extra hepatic bile duct. Histologic types, stage of disease, grade and survival rate. Cancer 70: 1498-1501, 1992.

2. Henson DE, Albores-Saaverdra J and Corle D: Carcinoma of the gallbladder. Histologic types, stage of disease, grade, and survival rate. Cancer 70: 1498-1497,1992.

3. de Groen PC, Gores GJ, LaRusso NF, et al: Biliary tract cancers. N Engl J Med 341: 1368-1378, 1999.

4. Tavani A, Negri E and La Vecchia C: Biliary tract tumors. Ann Ist Super Sanita 32: 615-619, 1996.

5. Nimura Y: Extented surgery in bilio-pancreatic cancer: the Japanese experience. Semin Oncol 29: 17-22, 2002.

6. Falkson G, MacIntyre JM and Moertel CG: Eastern Cooperated Oncology Group experience with chemotherapy for inoperable gallbladder and bile duct cancer. Cancer 54: 965-969, 1984.

7. Yee K, Sheppard BC, Domreis J and Blanke CD: Cancers of the gallbladder and biliary ducts. Oncology 16: 939-949, 2002 .

8. Ellis PA, Norman A, Hill A, et al: Epirubicin, cisplatin, and infusional 5-fluorouracil (5-FU) (ECF) in hepatobiliary tumours. Eur J Cancer 31: 1594-1598, 1995.

9. Sanz-Altamimra PM, Ferrante K, Jenkins RL, et al: A phase II trial of 5-fluorouracil, leucovorin, and carboplatin in patients with unresectable biliary tree carcinoma. Cancer 82: 2321-2325, 1998.

10. Gebbia V, Giuliani F, Maiello E, et al: Treatment of inoperable and/or metastatic biliary tree carcinomas with single-agent gemcitabine or in combination with levofolinic acid and infusional fluorouracil: results of multicenter phase II study. J Clin Oncol 19: 4089-4091, 2001.

11. Pollera CF, Ceribelli A, Crecco M, et al: Weekly gemcitabine in advanced bladder cancer: a preliminary report from a phase I study. Ann Oncol 5: 182-184, 1994.

12. Pasetto LM, D'Andrea MR, Falci C and Monfardini S: Gemcitabine in advanced biliary tract cancers. Crit Rev Oncol Hematol 61: 231-242, 2007.

13. Bergman AM, Pinedo HM and Peters GJ: Determinants of resistance to 2',2'-difluorodeoxycytidine (gemcitabine). Drug Resist Updat 5: 19-33, 2002.

14. Galmarni CM, Clarke ML, Falette N, et al: Expression of a nonfunctional p53 affects the sensitivity of cancer cells to gemcitabine. Int J Cancer 97: 439-445, 2002. 
15. Shi X, Liu S, Kleeff J, Friess N and Buchler MW: Acquired resistance of pancreatic cancer cells towards 5-FU and gemcitabine is associated with altered expression of apoptosisregulating genes. Oncology 62: 354-362, 2002.

16. Duxbury MS, Ito $\mathrm{H}$, Zinner MJ, et al: Inhibition of SRC tyrosine kinase impairs inherent and acquired gemcitabine resistance in human pancreatic adenocarcinoma cells. Clin Cancer Res 10: 2307-2318, 2004.

17. Akada M, Crnogorac-Jurcevic T, Lattimore S, et al: Intrinsic chemoresistance to gemcitabine is associated with decreased expression of BNIP3 in pancreatic cancer. Clin Cancer Res 11: 3094-3101, 2005

18. Duxbury MS, Ito H, Benoit E, et al: RNA interference targeting focal adhesion kinase enhances pancreatic adenocarcinoma gemcitabine chemosensitivity. Biochem Biophys Res Commun 311: 786-792, 2003.

19. Galmarini CM, Clarke ML, Jordheim L, et al: Resistance to gemcitabine in a human follicular lymphoma cell line is due to partial deletion of the deoxycytidine kinase gene. BMC Pharmacol 4: 8, 2004.

20. Eliopoulos N, Cournoyer D and Momparler RL: Drug resistance to 5-aza-2'-deoxycytidine, 2',2'-difluorodeoxycytidine, and cytosine arabinoside conferred by retroviral-mediated transfer of human cytidine deaminase cDNA into murine cells. Cancer Chemother Pharmacol 42: 373-378, 1998.

21. Davidsons JD, Ma L, Flagella M, et al: An increase in the expression of ribonucleotide reductase large subunit 1 is associated with gemcitabine resistance in non-small cell lung cancer cell lines. Cancer Res 64: 3761-3766, 2004.

22. Bergman AM, Ejik PP, Ruiz van Haperen VW, et al: In vivo induction of resistance to gemcitabine results in increased expression of ribonucleotide reductase subunit M1 as the major determinant. Cancer Res 65: 9510-9516, 2005.

23. Nakahira S, Nakamori S, Tsujie M, et al: Involvement of ribonucleotide reductase M1 subunit overexpression in gemcitabine resistance of human pancreatic cancer. Int J Cancer 120: 1355-1363, 2007

24. Dumontet C, Bauchu EC, Fabianowska K, et al: Common resistance mechanisms to nucleotide analogues in variants of the human erythroleukemic line K562. Adv Exp Med Biol 457: 571-577, 1999.

25. Duxbury MS, Ito H, Zinner MJ, et al: RNA interference targeting the M2 subunit of ribonucleotide reductase enhances pancreatic adenocarcinoma chemosensitivity to gemcitabine. Oncogene 23: 1539-1548, 2004.

26. Jordan A and Reichard P: Ribonucleotide reductase. Ann Rev Biochem 67: 71-98, 1998.
27. Xue L, Zhou B, Liu X, et al: Wild-type p53 regulates human ribonucleotide reductase by protein-protein interaction with p53R2 as well as hRRM2 subunits. Cancer Res 63: 980-986, 2003.

28. Wright JA, Chan AK, Choy BK, et al: Regulation and drug resistance mechanisms of mammalian ribonucleotide reductase, and the significance to DNA synthesis. Biochem Cell Biol 68: 1364-1371, 1990

29. Hurta RA and Wright JA: Alterations in the activity and regulation of mammalian ribonucleotide reductase by chlorambucil, a DNA damaging agent. J Biol Chem 267: 7066-7071, 1992 .

30. Heinemann V, Hertel LW, Grindey GB and Plunkett W: Comparison of the cellular pharmacokinetics and toxicity of 2 ', 2'-difluorodeoxycytidine and 1- $\beta$-D-arabinofuranosylcytocine. Cancer Res 48: 4024-4031, 1998.

31. Plunkett W, Huang P, Xu Y-Z, et al: Metabolism, mechanisms of action, and self-potentiation. Semin Oncol 22: 3-9, 1995.

32. Eriksson S and Martin DW Jr: Ribonucleotide reductase in cultured mouse lymphoma cells. Cell cycle-dependent variation in the activity of subunit protein M2. J Biol Chem 256: 9436-9440, 1981

33. Guittet O, Hakansson P, Voevodskaya N, et al: Mammalian $\mathrm{p} 53 \mathrm{R} 2$ protein forms an active ribonucleotide reductase in vitro with the R1 protein, which is expressed both in resting cells in response to DNA damage and in proliferating cells. J Biol Chem 276: 40647-40651, 2001.

34. Jiao W, Kitajima Y, Ogawa A and Miyazaki K: Establishment and characterization of human hilar bile duct carcinoma cell line and cell stein. J Hepatobiliary Pancreat Surg 7: 417-425, 2000.

35. Therasse P, Arbuck SG, Eisenhauer EA, et al: New guidelines to evaluate the response to treatment in solid tumor. European organization for reseach and treatment of cancer, National Cancer Institute of the United States, National Cancer Institute of Canada. J Natl Cancer Inst 92: 205-216, 2000.

36. Jordheim LP, Guittet O, Lepoivre M, et al: Increased expression of the large subunit of ribonucleotide reductase is involved in resistance to gemcitabine in human mammary adenocarcinoma cels. Mol Cancer Ther 4: 1268-1276, 2005.

37. Cao MY, Lee Y, Feng NP, et al: Adenovirus-mediated ribonucleotide reductase R1 gene therapy of human colon adenocarcinoma. Clin Cancer Res 9: 4553-4561, 2003.

38. Hertel LW, Boder GB, Kroin JS, et al: Evaluation of the antitumor activity of gemcitabine (2',2'-difluoro-2'-deoxycytidine). Cancer Res 50: 4417-4422, 1990. 